Pacific Journal of Mathematics

A NOTE ON DERIVATIONS WITH POWER CENTRAL VALUES 


\title{
A NOTE ON DERIVATIONS WITH POWER CENTRAL VALUES ON A LIE IDEAL
}

\author{
JEFFREY BERGEN AND LUISA CARINI
}

\begin{abstract}
Let $R$ be a prime ring of characteristic $\neq 2$ with a derivation $d \neq 0$ and a non-central Lie ideal $U$ such that $d(u)^{n}$ is central, for all $u \in U$. We prove that $R$ must satisfy $s_{4}$, the standard identity in 4 variables; hence $R$ is either commutative or an order in a 4-dimensional simple algebra. This result extends a theorem of Herstein to Lie ideals.
\end{abstract}

In [2] Herstein shows that if $R$ is a prime ring with center $Z$ and if $d \neq 0$ is a derivation of $R$ such that $d(x)^{n} \in Z$, for all $x \in R$, then $R$ satisfies $s_{4}$, the standard identity in 4 variables. This theorem indicates that the global structure of a ring is often tightly connected to the behaviour of one of its derivations. The purpose of this note is to show that the same conclusion holds for prime rings of characteristic $\neq 2$ even if we assume only that $d(u)^{n}$ is central for those $u$ in some non-central Lie ideal.

We will proceed by first proving the result when $d$ is inner. We will then use Kharchenko's theorem on differential identities [5] to reduce to the case where $d$ is inner on the Martindale quotient ring of $R$. By using Kharchenko's theorem, our proof will actually be somewhat simpler than the proof in [2].

In all that follows, unless stated otherwise, $R$ will be a prime ring of characteristic $\neq 2, U$ a non-central Lie ideal of $R, d \neq 0$ a derivation of $R$, and $n \geq 1$ a fixed integer such that $d(u)^{n}$ is central, for all $u \in U$. For any ring $S, Z=Z(S)$ will denote its center. For subsets $A, B \subset R,[A, B]$ will be the additive subgroup generated by all $[a, b]=a b-b a ; a \in A$, $b \in B$. In addition, $s_{4}$ will denote the standard identity in 4 variables.

By a result of Herstein [3], $U \supset[I, R]$ for some $I \neq 0$, an ideal of $R$. Therefore, we will assume throughout that $U \supset[I, R]$.

We will also make frequent and important use of the following three results. We do not necessarily state them in their fullest generality.

1. (Carini-Giambruno [1].) If $U \not \subset Z(R)$ is a Lie ideal of a prime ring $R$ of characteristic $\neq 2$, and if $d$ is a derivation of $R$ such that $d(u)^{n}=0$, for all $u \in U$, then $d=0$. 
2. (Kharchenko [5].) If $d$ is a derivation of a prime ring $R$ and if there exist $a_{i}, b_{i}, c_{i}, e_{i} \in R$ such that $\sum a_{i} d(x) b_{i}=\sum c_{i} x e_{i}$, for all $x$ in a non-zero ideal, then either $d$ is inner in the Martindale quotient ring of $R$ or $\sum a_{i} x b_{i}=\sum c_{i} x e_{i}=0$ for all $x$ in the ideal.

3. (Herstein-Procesi-Schacher [4].) If $R$ is a prime ring satisfying a polynomial identity and if for all $x, y \in R$ there exists an $n=n(x, y) \geq 1$ such that $[x, y]^{n} \in Z(R)$, then $R$ satisfies $s_{4}$.

We now begin the work necessary to prove our theorem with

LEMMA 1. If $J \neq 0$ is an ideal of $R$ then $J \cap Z(R) \neq 0$. Furthermore, $R_{Z}$, the localization of $R$ at $Z(R)$, is simple with 1 .

Proof. Let $V=\left[I, J^{2}\right]$; it is easy to check that $V$ is a non-central Lie ideal of $R$ and $V \subset U$. Since $d\left(J^{2}\right) \subset J d(J)+d(J) J \subset J$, we have

$$
d(V)=d\left(\left[I, J^{2}\right]\right) \subset\left[I, d\left(J^{2}\right)\right]+\left[d(I), J^{2}\right] \subset J .
$$

By the result of Carini and Giambruno [1], there is some $v \in V$, such that $d(v)^{n} \neq 0$.

Hence $0 \neq d(v)^{n} \in J \cap Z(R)$.

If $K \neq 0$ is an ideal of $R_{Z}$, then $K \cap R$ is a non-zero ideal of $R$; hence $(K \cap R) \cap Z(R) \neq 0$. Therefore $K$ contains invertible elements of $R_{Z}$ and so, $R_{Z}$ is simple with 1 .

We can now prove the special case of our result when $d$ is an inner derivation.

THEOREM 2. Let $R$ be a prime ring of characteristic $\neq 2$ and let $a \in R$, $a \notin Z(R)$ be such that $(a u-u a)^{n} \in Z(R)$, for all $u \in U$, where $U \not \subset Z(R)$ is a Lie ideal of $R$. Then $R$ satisfies $s_{4}$.

Proof. By Lemma 1 we may localize $R$ at $Z(R)$, and it follows that $(a u-u a)^{n} \in Z\left(R_{Z}\right)$, for all $u \in\left[R_{Z}, R_{Z}\right]$. Therefore in order to prove that $R$ satisfies $s_{4}$, we may assume that $R$ is simple with 1 and $U \supset[R, R]$. Since $a \notin Z,\left[(a(x y-y x)-(x y-y x) a)^{n}, z\right]$ is a non-trivial generalized polynomial identity for $R$. Thus, by a result of Martindale [6], $R$ is primitive with minimal right ideal, whose commuting ring $D$ is a division ring finite dimensional over $Z(R)$. However, since $R$ is simple with $1, R$ must be artinian; thus $R=D_{k}$ for some $k \geq 1$.

If $Z(R)$ is infinite, let $F$ be a maximal subfield of $D$ and consider $\bar{R}=R \otimes_{Z(R)} F=F_{m}$, for some $m \geq 1$. 
A Vandermonde determinant argument shows that in $\bar{R}$, $\left[(a(x y-y x)-(x y-y x) a)^{n}, z\right]$ is still an identity. If $Z(R)$ is finite then $D$ is a finite division ring; hence $D=Z(R)$. In either case, to prove that $R$ satisfies $s_{4}$ it is enough to consider the case where $R=Z(R)_{m}$, for some $m \geq 1$, and $(a(x y-y x)-(x y-y x) a)^{n} \in Z(R)$ for all $x, y \in R$.

$R$ is now the ring of linear transformations over $Z(R)$ of an $m$-dimensional vector space $V$. Since $a \notin Z(R)$, we can find some $v \in V$ such that $v$ and $v a$ are linearly independent.

It suffices to show that $m \leq 2$, so we suppose not and let $t_{1}, t_{2}, \ldots, t_{m-2} \in V$ be such that $T=\left\{v, v a, t_{1}, \ldots, t_{m-2}\right\}$ is a basis for $V$ over $Z(R)$.

Let $x \in R$ such that vax $=t_{1}$ and $t x=0$, for all other $t \in T$. In addition, let $y \in R$ be such that $t_{1} y=v$ and $t y=0$, for all other $t \in T$. Therefore

$$
\begin{aligned}
& v(a(x y-y x)-(x y-y x) a)=v \quad \text { and so, } \\
& v(a(x y-y x)-(x y-y x) a)^{n}=v .
\end{aligned}
$$

Hence $(a(x y-y x)-(x y-y x) a)^{n}$ is a non-zero element of $Z(R)$. Thus $m$ equals the rank of $(a(x y-y x)-(x y-y x) a)^{n}$ as a linear transformation. However, $x y-y x$ has rank 1 ; therefore $a(x y-y x)-$ $(x y-y x) a$ has rank $\leq 2$ and so, $(a(x y-y x)-(x y-y x) a)^{n}$ also has rank $\leq 2$.

Therefore $m \leq 2$ and we have proved that $R$ must satisfy $s_{4}$.

At this point we would like to reduce the general case down to the case where $d$ is inner. To do this, in addition to using Kharchenko's theorem [5], we need the following lemma which is similar to Lemma 4 of [2].

LEMMA 3. If $\operatorname{char} R=p>0$ and if $d(Z) \neq 0$, then $R$ satisfies $s_{4}$.

Proof. Let $\gamma \in Z$ be such that $d(\gamma) \neq 0$ and let $K=\{\alpha \in Z \mid d(\alpha)$ $=0\}$. Since char $R=p>0$, for every $\beta \in Z$ we have $\beta^{p} \in K$. Now, if $K$ were finite, then $K$ would be a field and the quotient field of $Z$ would be algebraic over $K$. Therefore there would exist an integer $m \geq 1$ such that $\gamma^{p^{m}}=\gamma$, resulting in the contradiction $0=d\left(\gamma^{p^{m}}\right)=d(\gamma)$. Hence $K$ is infinite.

In $R_{Z}$, let $T=\{d(\gamma) /(\alpha+\gamma) \mid \alpha \in K\}$; by the preceding argument, $T$ is an infinite subset of $Z\left(R_{Z}\right)$. If $u \in[I, R]$ we note that $(\alpha+\gamma) u \in$ $[I, R]$, for all $\alpha \in K$. Hence

$$
d((\alpha+\gamma) u)^{n}=(d(\gamma) u+(\alpha+\gamma) d(u))^{n} \in Z(R)
$$


for all $u \in[I, R]$ and $\alpha \in K$. Dividing by $(\alpha+\gamma)^{n}$ results in $(\lambda u+d(u))^{n} \in Z\left(R_{Z}\right)$, for all $\lambda \in T$ and $u \in[I, R]$. We again use a Vandermonde determinant argument, since $T$ is infinite, to see that $u^{n} \in Z\left(R_{Z}\right) \cap R=Z(R)$.

Thus $[i, r]^{n} \in Z(R)$, for all $i \in I, r \in R$; which implies that $R$ satisfies a polynomial identity and, after localizing at $Z(R)$, we have $[x, y]^{n} \in Z\left(R_{Z}\right)$, for all $x, y \in R_{Z}$.

By the result of Herstein-Procesi-Schacher [4], $R_{Z}$ satisfies $s_{4}$, hence $R$ satisfies $s_{4}$.

We can now prove the main result of this note.

THEOREM 4. Let $R$ be a prime ring of characteristic $\neq 2$ with a derivation $d \neq 0$ and a Lie ideal $U \not \subset Z(R)$ such that $d(u)^{n} \in Z(R)$, for all $u \in U$. Then $R$ satisfies $s_{4}$; hence $R$ is commutative or an order in a 4 dimensional simple algebra.

Proof. Suppose $d$ is inner in the Martindale quotient ring of $R$ and is induced by some element $q$. However, by Lemma 1 , the Martindale quotient ring of $R$ is $R_{Z}$; hence $q=a / \alpha$, for some $a \in R, \alpha \in Z(R)$. Therefore the inner derivation of $R$ induced by $a$ satisfies all the hypotheses of Theorem 2, thus in this case, $R$ satisfies $s_{4}$.

As a result, it now suffices to show that $d$ is inner in the Martindale quotient ring of $R$. Let $x_{1}, \ldots, x_{n} \in I$ and $y_{1}, \ldots, y_{n} \in R$ and let $z_{l}=$ $\left[x_{i}, y_{i}\right]$.

By hypothesis $d\left(z_{1}+\cdots+z_{n}\right)^{n} \in Z(R)$ and, by linearization, we obtain

$$
\sum_{\pi \in S_{n}} d\left(z_{\pi(1)}\right) \cdots d\left(z_{\pi(n)}\right) \in Z(R)
$$

where $S_{n}$ denotes the symmetric group in $n$ letters.

Expanding each $d\left(z_{l}\right)$ we obtain

$$
\begin{aligned}
& \sum_{\pi \in S_{n}}( {\left.\left[d\left(x_{\pi(1)}\right), y_{\pi(1)}\right]+\left[x_{\pi(1)}, d\left(y_{\pi(1)}\right)\right]\right) \cdots } \\
&\left(\left[d\left(x_{\pi(n)}\right), y_{\pi(n)}\right]+\left[x_{\pi(n)}, d\left(y_{\pi(n)}\right)\right]\right) \in Z(R) .
\end{aligned}
$$

If we multiply out the terms of $(*)$ we will obtain a sum of terms all of whom mention $y_{1}$ or $d\left(y_{1}\right)$ exactly once. Therefore, after commuting $(*)$ with any $r \in R$, we obtain an expression of the form

$$
\sum a_{l} y_{1} b_{\imath}+\sum c_{i} d\left(y_{1}\right) e_{\imath}=0
$$

where $a_{i}, b_{l}, c_{l}, e_{l}$ are products obtained from $r$ and the $x_{l}, d\left(x_{t}\right), y_{l}$, and $d\left(y_{1}\right)$, but not $y_{1}$ or $d\left(y_{1}\right)$. By Kharchenko's result [5], if $d$ is not 
inner in the Martindale quotient ring then

$$
\sum a_{i} y_{1} b_{i}+\sum c_{i} y_{1} e_{i}=0, \text { for all } y_{1} \in R .
$$

Thus in $(*)$ we may replace all occurrences of $d\left(y_{1}\right)$ by $y_{1}$. Similarly, we may sequentially replace each $d\left(y_{1}\right)$ by $y_{i}$ and then each $d\left(x_{1}\right)$ by $x_{i}$ to finally obtain

$$
2^{n} \sum_{\pi \in S_{n}}\left[x_{\pi(1)}, y_{\pi(1)}\right] \cdots\left[x_{\pi(n)}, y_{\pi(n)}\right] \in Z(R) .
$$

Since $\operatorname{char} R \neq 2, R$ satisfies a polynomial identity. However, by Lemma 3, if char $R=p>0$ and if $R$ fails to satisfy $s_{4}$, then $d(Z)=0$.

But now, by the Skolem-Noether theorem, $d$ is inner on $R_{Z}$ since $d\left(Z\left(R_{Z}\right)\right)=0$. Thus in this case the proof is complete.

Finally, if $\operatorname{char} R=0$ then let $x_{1}=x_{2}=\cdots=x_{n}$ and $y_{1}=y_{2}=$ $\cdots=y_{n}$; therefore $(* *)$ becomes $2^{n} n !\left[x_{1}, y_{1}\right]^{n} \in Z(R)$ and so, $\left[x_{1}, y_{1}\right]^{n}$ $\in Z(R)$. As in the proof of Lemma 3 , by [4], $R$ satisfies $s_{4}$, thereby completing the proof.

\section{REFERENCES}

[1] L. Carini and A. Giambruno, Lie ideals and nil derivations, Bollettino U.M.I., (6) 4-A (1985), 497-503.

[2] I. N. Herstein, Derivations of prime rings having power central values, Algebraist's Homage, Contemporary Mathematics Vol. 13, A.M.S., Providence, Rhode Island, 1982.

[3] On the Lie structure of an associative ring, J. Algebra, 14 (1970), 561-571.

[4] I. N. Herstein, C. Procesi and M. Schacher, Algebraic valued functions on non commutative rings, J. Algebra, 36 (1975), 128-150.

[5] V. K. Kharchenko, Differential identities of prime rings, Algebra i Logika, 17 (1978), 220-238.

[6] W. S. Martindale III, Prime rings satisfying a generalized polynomial identity, J. Algebra, 12 (1969), 576-584.

Received January 15, 1987. First author's research supported, in part, by a grant from the University Research Council at De Paul University. Second author's research supported by a grant from M.P.I.

De Paul University

CHICAGO, IL 60614

U.S.A.

AND

DELI'UNIVERSITA

VIA C. BATTISTI N ${ }^{\circ} 90$

98100 MESSINA, ITALY 



\title{
PACIFIC JOURNAL OF MATHEMATICS EDITORS
}

\author{
V. S. VARADARAJAN \\ (Managing Editor) \\ University of California \\ Los Angeles, CA 90024 \\ HERBERT Clemens \\ University of Utah \\ Salt Lake City, UT 84112 \\ R. FINN \\ Stanford University \\ Stanford, CA 94305
}

\author{
HERMANN FLASCHKA \\ University of Arizona \\ Tucson, AZ 85721
}

Ramesh A. Gangolli University of Washington Seattle, WA 98195

VAUGHAN F. R. JONES University of California Berkeley, CA 94720
ROBION KIRBY

University of California

Berkeley, CA 94720

C. C. MOORE

University of California

Berkeley, CA 94720

HAROLD STARK

University of California, San Diego La Jolla, CA 92093

\section{ASSOCIATE EDITORS}

\author{
R. ARENS \\ E. F. BECKENBACH \\ B. H. NEUMANN \\ F. WOLF \\ K. YOSHIDA \\ (1906-1982)

\section{SUPPORTING INSTITUTIONS}

\section{UNIVERSITY OF ARIZONA} \\ UNIVERSITY OF BRITISH COLUMBIA \\ CALIFORNIA INSTITUTE OF TECHNOLOGY \\ UNIVERSITY OF CALIFORNIA \\ MONTANA STATE UNIVERSITY \\ UNIVERSITY OF NEVADA, RENO \\ NEW MEXICO STATE UNIVERSITY \\ OREGON STATE UNIVERSITY \\ UNIVERSITY OF OREGON \\ UNIVERSITY OF SOUTHERN CALIFORNIA \\ STANFORD UNIVERSITY \\ UNIVERSITY OF HAWAII \\ UNIVERSITY OF TOKYO \\ UNIVERSITY OF UTAH \\ WASHINGTON STATE UNIVERSITY \\ UNIVERSITY OF WASHINGTON
}

The Supporting Institutions listed above contribute to the cost of publication of this Journal, but they are not owners or publishers and have no responsibility for its content or policies.

Mathematical papers intended for publication in the Pacific Journal of Mathematics should be in typed form or offset-reproduced (not dittoed), double spaced with large margins. Please do not use built up fractions in the text of the manuscript. However, you may use them in the displayed equations. Underline Greek letters in red, German in green, and script in blue. The first paragraph must be capable of being used separately as a synopsis of the entire paper. In particular it should contain no bibliographic references. Please propose a heading for the odd numbered pages of less than 35 characters. Manuscripts, in triplicate, may be sent to any one of the editors. Please classify according to the scheme of Math. Reviews, Index to Vol. 39. Supply name and address of author to whom proofs should be sent. All other communications should be addressed to the managing editor, or Elaine Barth, University of California, Los Angeles, California 90024

There are page-charges associated with articles appearing in the Pacific Journal of Mathematics. These charges are expected to be paid by the author's University, Government Agency or Company. If the author or authors do not have access to such Institutional support these charges are waived. Single authors will receive 50 free reprints; joint authors will receive a total of 100 free reprints. Additional copies may be obtained at cost in multiples of 50 .

The Pacific Journal of Mathematics is issued monthly as of January 1966. Regular subscription rate: $\$ 190.00$ a year (5 Vols., 10 issues). Special rate: $\$ 95.00$ a year to individual members of supporting institutions.

Subscriptions, orders for numbers issued in the last three calendar years, and changes of address should be sent to Pacific Journal of Mathematics, P.O. Box 969, Carmel Valley, CA 93924, U.S.A. Old back numbers obtainable from Kraus Periodicals Co., Route 100, Millwood, NY 10546.

The Pacific Journal of Mathematics at P.O. Box 969, Carmel Valley, CA 93924 (ISSN 0030-8730) publishes 5 volumes per year. Application to mail at Second-class postage rates is pending at Carmel Valley, California, and additional mailing offices. Postmaster: send address changes to Pacific Journal of Mathematics, P.O. Box 969, Carmel Valley, CA 93924.

PUBLISHED BY PACIFIC JOURNAL OF MATHEMATICS, A NON-PROFIT CORPORATION Copyright (C) 1988 by Pacific Journal of Mathematics 


\section{Pacific Journal of Mathematics}

\section{Vol. 132, No. $2 \quad$ February, 1988}

Jeffery Marc Bergen and Luisa Carini, A note on derivations with power central values on a Lie ideal ..............................209

Alfonso Castro and Sumalee Unsurangsie, A semilinear wave equation

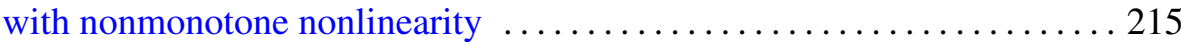

Marius Dadarlat, On homomorphisms of matrix algebras of continuous

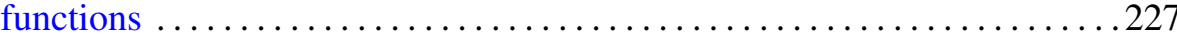

A. Didierjean, Quelques classes de cobordisme non orienté refusant de se

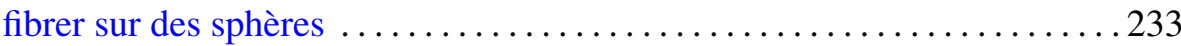

Edward George Effros and Zhong-Jin Ruan, On matricially normed spaces

Sherif El-Helaly and Taqdir Husain, Orthogonal bases are Schauder bases

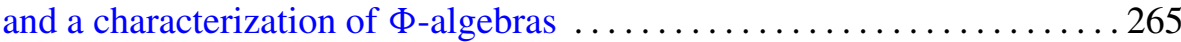

Edward Richard Fadell and Peter N-S Wong, On deforming $G$-maps to be

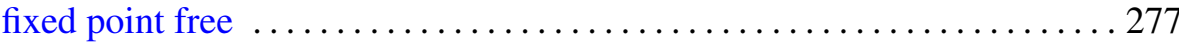

Jean-Jacques Gervais, Stability of unfoldings in the context of equivariant

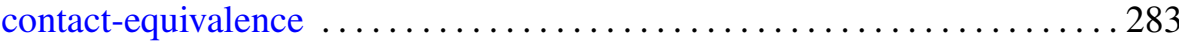

Douglas Martin Grenier, Fundamental domains for the general linear group

Ronald Scott Irving and Brad Shelton, Loewy series and simple projective

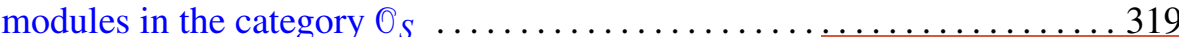

Russell Allan Johnson, On the Sato-Segal-Wilson solutions of the K-dV equation

Thomas Alan Keagy and William F. Ford, Acceleration by subsequence transformations

Min Ho Lee, Mixed cusp forms and holomorphic forms on elliptic varieties

Charles Livingston, Indecomposable surfaces in 4-space 